\title{
End of Life Problem Identified
}

National Cancer Institute

\section{Source}

National Cancer Institute. End of Life Problem Identified. NCI Thesaurus. Code C92048.

Problems traced to the device reaching the end of its useful life. 